\title{
ON CONTINUITY OF FIXED POINTS OF COLLECTIVELY CONDENSING MAPS
}

\author{
MARTIN FAN CHENG
}

\begin{abstract}
In this paper, we prove, in two parts, the following claim. Let $X$ be a Banach space and $\Lambda$ an arbitrary topological space. Suppose that $T: \Lambda \times X \rightarrow X$ is collectively condensing; then the fixed point set $S(\lambda, y)$ has closed graph if and only if $T$ is continuous in both $\lambda$ and $y$.
\end{abstract}

\section{Zvi Artstein [1] proved}

TheOrem 0. Suppose $X$ is a Banach space and $\Lambda$ a metric space. Let $T: \Lambda \times X \rightarrow X$ be collectively condensing, i.e., for every $B \subset X$,

$$
\chi\left(\bigcup_{\lambda \in \Lambda} T(\lambda, B)\right) \leqslant \chi(B),
$$

where equality implies $\chi(B)=0$ and $\chi$ is the Kuratowski measure of noncompactness, defined as $\chi(B)=\inf \{d \mid B$ can be covered by a finite number of subsets of diameter $<d\}$. Let $S(\lambda, y)=\{x \in X \mid x=T(\lambda, x)+y\}$. Then, $S(\lambda, y)$ is upper-semicontinuous if and only if $T(\lambda, x)$ is continuous in $\lambda$ and $x$ simultaneously.

At the end of his paper, he posed an open question. Is Theorem 0 still true if $\Lambda$ is a general topological space? Upon investigation we find that $S(\lambda, y)$ has closed graph if and only if $T(\lambda, x)$ is continuous in both $\lambda$ and $x$.

The following definitions are intended to refresh the memory of the readers.

Definition 0.1. Let $E_{1}$ be a topological space, and $\Lambda$ a subset of a topological space $E_{2}$. We say a multifunction $F: \Lambda \rightarrow E_{1}$ is upper-semicontinuous at $\lambda_{0} \in \Lambda$ if for each open set $G$ in $E_{1}$ containing $F\left(\lambda_{0}\right)$, there exists an open neighborhood $U\left(\lambda_{0}\right)$ of $\lambda_{0}$ in $E_{2}$ such that $F\left(U\left(\lambda_{0}\right) \cap \Lambda\right) \subset G$.

Definition 0.2. $F$ is upper-semicontinuous on $\Lambda$ if it is upper-semicontinuous at each $\lambda_{0} \in \Lambda$.

To prove our main theorem, we need the following tools.

TheOREM 0.3 [2, THEOREM 11.5]. A net has $y$ as a cluster point iff it has a subnet which converges to $y$.

Theorem 0.4 [2, Theorem 11.8]. Let $f: X \rightarrow Y$. Then $f$ is continuous at $x_{0} \in X$ iff whenever $x_{\lambda} \rightarrow x_{0}$ in $X$, then $f\left(x_{\lambda}\right) \rightarrow f\left(x_{0}\right)$ in $Y$.

Received by the editors March 22, 1976.

AMS (MOS) subject classifications (1970). Primary 47H10; Secondary 54H25.

Key words and phrases. Collective condensing, closed graph, upper-semicontinuity. 
THEOREM 0.5 [2, THEOREM 11Dc]. If every subnet of a net $\left(x_{\lambda}\right)$ has a subnet converging to $x$, then $\left(x_{\lambda}\right)$ converges to $x$.

The following are our main theorems.

TheOREM 1. Let $X$ be a Banach space, and $\Lambda$ a topological space. Let $T: \Lambda \times X \rightarrow X$ be collectively condensing. Suppose that $S$ is upper-semicontinuous; then $T$ is continuous in $\lambda$ and $x$.

Proof. We will denote $T(\lambda, \cdot)$ by $T_{\lambda}$. Let $\left(\lambda_{\alpha}, x_{\alpha}\right)$ be a net in $\Lambda \times X$ converging to $\left(\lambda_{0}, x_{0}\right)$. We will show that there exists a subnet $\left(T_{\lambda_{\alpha_{\omega}}} x_{\alpha_{\omega}}\right)$ converging to $\left(T_{\lambda_{0}} x_{0}\right)$. Let $r_{n}=1 / 2^{n}, n=1,2, \ldots$ Let $\sigma_{1}=\left\{x_{\alpha} \mid\left\|x_{\alpha}-x_{0}\right\|\right.$ $\left.\leqslant r_{1}\right\}$. We claim $\mathscr{T}_{1}$ is a subnet of $\left(x_{\alpha}\right)$. Indeed, a routine argument shows that the set $A_{1}=\left\{\alpha \mid x_{\alpha} \in \sigma_{1}\right\}$ is a directed set, cofinal with the given directed set. Consequently, $\left\{\left(\lambda_{\alpha}, x_{\alpha}\right) \mid \alpha \in A_{1}\right\}$ is a subnet, whereas $\sigma_{1}$ and $\left\{T_{\lambda_{\alpha}} x_{\alpha} \mid \alpha \in A_{1}\right\}$ are subnets of $\left(x_{\alpha}\right)$ and $\left(T_{\lambda_{\alpha}} x_{\alpha}\right)$, respectively.

Let $x_{\alpha}, x_{\beta} \in \mathcal{T}_{1}$. Then $\left\|x_{\alpha}-x_{\beta}\right\| \leqslant\left\|x_{\alpha}-x_{0}\right\|+\left\|x_{\beta}-x_{0}\right\| \leqslant 2 r_{1}$. Hence, $\operatorname{diam}\left(\sigma_{1}\right) \leqslant 2 r_{1}$. By collective condensingness, $\chi\left(\left\{T_{\lambda_{\alpha}}\left(x_{\alpha}\right) \mid \alpha \in A_{1}\right\}\right)$ $<\chi\left(\widetilde{T}_{1}\right) \leqslant 2 r_{1}$. Let $d_{1}=\chi\left(\left\{T_{\lambda_{\alpha}}\left(x_{\alpha}\right) \mid \alpha \in A_{1}\right\}\right)$. Choose $\varepsilon_{1}>0$ so that $d_{1}+\varepsilon_{1}$ $<2 r_{1}$. Then, there exists a finite cover: $S_{1}^{1}, S_{2}^{1}, \ldots, S_{k(1)}^{1}$ of $\delta_{1}=\left\{T_{\lambda_{\alpha}}\left(x_{\alpha}\right) \mid\right.$ $\left.\alpha \in A_{1}\right\}$. We claim that there is a set $S_{j}^{1}, 1 \leqslant j \leqslant k(1)$, which contains a subnet of $\delta_{1}$. The following is the proof of this claim. ${ }^{1}$ Let $\delta=\cup_{i=2}^{k(1)} S_{i}^{1}, A_{\delta}$ $=\left\{\alpha \in A_{1} \mid T_{\lambda_{\alpha}}\left(x_{\alpha}\right) \in \delta\right\}$, and $A_{S_{i}^{1}}=\left\{\alpha \in A_{1} \mid T_{\lambda_{\alpha}}\left(x_{\alpha}\right) \in S_{i}^{1}\right\}$. Then $\left\{S_{1}^{1}, \delta\right\}$ is a cover of $\delta_{1}$, and $A_{S_{1}^{1}} \cup A_{\S}=A_{1}$. Suppose that $S_{1}^{1}$ does not contain a subnet of $\delta_{1}$. Then there exists $\alpha \in A_{1}$ such that $d \geqslant \alpha$ implies that $d \notin A_{S_{1}^{1}}$. Let

$$
D=\left\{d \in A_{1} \mid d \geqslant \alpha \text { for some } \alpha \in A_{1}, \text { and if } \bar{d} \geqslant d \text {, then } \bar{d} \notin A_{S}\right\} .
$$

We will show that $D$ is a directed set cofinal with $A_{1}$. Clearly, $D \subseteq A_{\S}$. We need only show that if $\alpha \in A$, then there exists a $d \in D$ such that $d \geqslant \alpha$. Since $A_{1}$ is a directed set, it follows that there exists a $\bar{d} \in A_{1}$ such that $\bar{d} \geqslant \alpha$. Let $\bar{D}=\left\{\bar{d} \in A_{1} \mid \bar{d} \geqslant \alpha\right\}$. Clearly, $\bar{D}$ is a directed set cofinal with $A_{1}$. Since $S_{1}^{1}$ does not contain a subnet of $\delta_{1}$, we can find $d^{*} \in A_{1}, d^{*} \geqslant \alpha$ such that $\bar{d} \geqslant d^{*}$ implies $\bar{d} \notin A_{S_{1}^{1}}$. Hence, $d^{*} \in D$. Thus, $D$ is a directed set cofinal with $A_{1}$. This means that if $S_{1}^{1}$ does not contain a subnet, then $\delta$ must contain one. By repeating the argument a finite number of times we see that there exists $S_{j}^{1}, 1 \leqslant j \leqslant k(1)$, which contains a subnet of $\delta_{1}$. Let $S_{1}=S_{j}^{1}$. Let $\Re_{1}$ denote the subset contained in $S_{1}$. Similarly, we can define $\mathfrak{x}_{2}, \Re_{3}, \ldots$. Clearly, $\Re_{1} \supset \Re_{2} \supset \cdots$, where $\Re_{n}$ is of diameter $2 r_{n}=2 / 2^{n}=1 / 2^{n-1}, n$ $=1,2, \ldots$ Thus $\cap_{n=1}^{\infty} \bar{\Re}_{n}=x$ for some $x \in X$. Let $B_{r_{n}}(x)$ be an open ball in $X$ with center at $x$ and radius $r_{n}$. Then $B_{r_{n}}(x)$ contains a subnet of $\left(T_{\lambda_{\alpha}}\left(x_{\alpha}\right)\right)$. This can be seen as follows. Consider $B_{r_{n+1}}(x)$. By construction, there exists $T_{\lambda_{\alpha_{n+2}}}\left(x_{\alpha_{n+2}}\right) \in \Re_{n+2}$ such that $T_{\lambda_{\alpha_{n+2}}}\left(x_{\alpha_{n+2}}\right)^{n_{n+1}} \in B_{r_{n+1}}(x)$. Thus, for each $T_{\lambda_{\alpha}} x_{\alpha}$ $\in \mathfrak{x}_{n+2}$,

\footnotetext{
${ }^{1}$ The proof of this claim is essentially due to Jack Porter.
} 


$$
\left\|T_{\lambda_{\alpha}}\left(x_{\alpha}\right)-x\right\| \leqslant\left\|T_{\lambda_{\alpha}}\left(x_{\alpha}\right)-T_{\lambda_{\alpha_{n+2}}}\left(x_{\alpha_{n+2}}\right)\right\|+\left\|T_{\lambda_{\alpha_{n+2}}}\left(x_{\alpha_{n+2}}\right)-x\right\|<2 r_{n+1}=r_{n},
$$

i.e., $\Re_{n+2} \subset B_{r_{n}}(x)$. This means, in other words, $x$ is a cluster point of $\Re_{1}$. Hence, by Theorem 0.3 , there is a subnet $\mathscr{N}$ of $\mathfrak{X}_{1}$ which converges to $x$. Let $\Re=\left(T_{\lambda_{\alpha_{\omega}}}\left(x_{\alpha_{\omega}}\right)\right)$. Then, clearly, $\left(\left(\lambda_{\alpha_{\omega}}, x_{\alpha_{\omega}}\right)\right) \rightarrow\left(\lambda_{0}, x_{0}\right)$. Let $y=x_{0}-x$, and for each $\alpha_{\omega}$, let $y_{\alpha_{\omega}}=x_{\alpha_{\omega}}-T_{\lambda_{\alpha_{\omega}}}\left(x_{\alpha_{\omega}}\right)$. Then, $\left(y_{\alpha_{\omega}}\right) \rightarrow y$, for some $y \in X$. By hypothesis, $x_{0} \in S\left(\lambda_{0}, y\right)$, i.e., $x_{0}=T_{\lambda_{0}}\left(x_{0}\right)-y$. Thus, $x=T_{\lambda_{0}}\left(x_{0}\right)$. Hence, $\left(T_{\lambda_{\alpha_{\omega}}}\left(x_{\alpha_{\omega}}\right)\right) \rightarrow\left(T_{\lambda_{0}}\left(x_{0}\right)\right)$. This actually shows that for each subnet of $\left(T_{\lambda_{\alpha}} x_{\alpha}\right)$, there is a subsubnet converging to $T_{\lambda_{0}}\left(x_{0}\right)$. By Theorem 0.5, $\left(T_{\lambda_{\alpha}}\left(x_{\alpha}\right)\right)$ $\rightarrow T_{\lambda_{0}}\left(x_{0}\right)$. By Theorem $0.4, T$ is continuous in $(\lambda, x)$. This completes the proof of Theorem 1.

REMARK 1. The above theorem is still true if $S$ has closed graph instead. The proof is essentially the same.

TheORem 2. Let $X$ be a Banach space, and $\Lambda$ a topological space. Let $T: \Lambda \times X \rightarrow X$ be continuous. For each $(\lambda, y) \in \Lambda \times X$, let $S(\lambda, y)=\{x$ $\in X \mid x=T(\lambda, x)+y\}$. Then $S$ has closed graph.

Proof. Let $\left(\lambda_{\alpha}, y_{\alpha}\right)$ be a net converging to $\left(\lambda_{0}, y_{0}\right)$. For each $\alpha$ let $x_{\alpha}$ $\in S\left(\lambda_{\alpha}, y_{\alpha}\right)$, i.e., $x_{\alpha}=T\left(\lambda_{\alpha}, x_{\alpha}\right)+y_{\alpha}$. We will show that if $\bar{x}$ is a cluster point of $\left(x_{\alpha}\right)$, then $\bar{x} \in S\left(\lambda_{0}, y_{0}\right)$. Let $\left(\lambda_{\alpha_{\omega}}\right)$ be a subnet of $\left(x_{\alpha}\right)$ converging to $\bar{x}$. Then, clearly, $\left(\lambda_{\alpha_{\omega}}\right) \rightarrow \lambda_{0},\left(y_{\alpha_{\omega}}\right) \rightarrow y_{0}$. Hence, $\left(T\left(\lambda_{\alpha_{\omega}}, x_{\alpha_{\omega}}\right)\right)=\left(x_{\alpha_{\omega}}-y_{\alpha_{\omega}}\right)$ $\rightarrow \bar{x}-y_{0}$. By continuity, $\left(T\left(\lambda_{\alpha_{\omega}}, x_{\alpha_{\omega}}\right)\right) \rightarrow T\left(\lambda_{0}, \bar{x}\right)$. By Hausdorff property, $T\left(\lambda_{0}, \bar{x}\right)=\bar{x}-y_{0}$, i.e., $\bar{x}=T\left(\lambda_{0}, \bar{x}\right)+y_{0}$, or $\bar{x} \in S\left(\lambda_{0}, y_{0}\right)$. Hence, $S$ has closed graph.

REMARK 2. Fred S. Van Vleck proved that if $X$ is a Euclidean space, then the result of Theorem 0 is still valid no matter what space $\Lambda$ is. However, if $X$ is an arbitrary Banach space, our conjecture is that $S$ need not be uppersemicontinuous even though $T$ is continuous and collectively condensing.

\section{REFERENCES}

1. Zvi Artstein, On continuous dependence of fixed points of condensing maps, 1974 (preprint).

2. Stephen Willard, General topology, Addison-Wesley, Reading, Mass., 1970. MR 41 \#9173.

Department of Mathematics, University of Kansas, LaWrence, Kansas 66045 